\title{
Slave particles made real: Critical Fermi surface at a Mott transition in Bose-Fermi mixtures
}

\author{
Mott criticality and pseudogap in Bose-Fermi mixtures \\ arXiv:1205.4026, by E. Altman, E. Demler, and A. Rosch
}

\section{Recommended with a commentary by Matthias Vojta, TU Dresden, Germany}

The Mott metal-insulator transition of fermions is a long-standing open problem in the theory of correlated electrons. While important conceptual ideas were developed in the early works of Mott, Hubbard, Gutzwiller, Brinkman/Rice, and others, a reliable solution of a relevant microscopic model is difficult because of the strong-coupling nature of the problem. For the single-band Hubbard model, some progress has been made using the dynamical mean-field theory (DMFT) and its generalizations, but the nature of the zero-temperature Mott transition is not understood to date $[1,2]$.

Considering that the defining characteristic of the metallic phase is a sharp Fermi surface of quasiparticles, the most pressing question is about the fate and evolution of this Fermi surface near criticality. This is intimately connected to the nature of the Mott-insulating phase: For insulators with conventional magnetic order, the metal-insulator transition is equivalent to a transition to a band insulator, and the Fermi surface disappears via pockets shrinking to zero volume. In contrast, exotic behavior is expected if the Mott insulator realizes a non-magnetic spin-liquid state: It has been proposed that then a critical Fermi surface [3] may emerge at the transition, with power-law singularities in the spectral function and a suppressed low-energy density of states (dubbed pseudogap) akin to the behavior of Luttinger liquids in $d=1$ space dimension.

Clearly, such behavior is of immense experimental relevance: Tunable Mott transitions, with non-magnetic phases on the insulating side, have been found in certain organic conductors [4]. Moreover, cuprate high-temperature superconductors can be viewed as doped Mott insulators [5], and the nature of their pseudogap regime is still an open and heavily debated issue.

A class of theoretical approaches to the fermionic Mott transition utilizes slave-particle representations of electrons in terms of auxiliary particles carrying fractionalized quantum numbers [6]. In the simplest case, these are charge-neutral spin- $1 / 2$ spinons, $f_{i \sigma}$, and spinless charge- $e$ holons, $b_{i}$, such that the physical electron operator reads $c_{i \sigma}^{\dagger}=f_{i \sigma}^{\dagger} b_{i}$ where $i$ is a site index. In a full description, these slave particles are coupled to emergent gauge fields. Unfortunately, the resulting theories are often hard to control, such that it remains unclear whether certain slave particles constitute true elementary excitations of the system.

In a recent theory paper [7], Altman and co-workers discovered a new playground for the described set of problems. They identified a tractable model for a non-trivial Mott transition in $d=3$, which features both a quantum critical Fermi surface and a pseudogap. They consider 
a Hubbard-like model of fermions and bosons [8], which can be realized using ultracold atoms on an optical lattice:

$$
\mathcal{H}=-t_{b} \sum_{\langle i j\rangle}\left(b_{i}^{\dagger} b_{j}+\text { H.c. }\right)-t_{f} \sum_{\langle i j\rangle}\left(f_{i}^{\dagger} f_{j}+\text { H.c. }\right)+\frac{1}{2} U_{b b} \sum_{i} n_{b i}\left(n_{b i}-1\right)+U_{b f} \sum_{i} n_{b i} n_{f i} .
$$

For simplicity, the fermions are assumed to be spinless.

A mixed Mott phase arises for total integer filling and large interactions, $U_{b f} \gg t_{f}, t_{b}$ and $U_{b b} \gg t_{b}$; the following discussion will be for $\left\langle n_{b i}\right\rangle+\left\langle n_{f i}\right\rangle=1$. Quite remarkably, second-order hopping processes in this Mott state allow for a site exchange of bosons and fermions, such that bound states, $c_{i}^{\dagger}=f_{i}^{\dagger} b_{i}$, become mobile particles. Under quite general conditions, the Mott insulator, while incompressible w.r.t. the original $c$ and $f$ particles, is then a Fermi liquid of $c$ bound states, with a true Fermi surface.

The Mott insulator can now be melt in two different ways, by reducing either $U_{b f}$ or $U_{b b}$. The first case has been studied in a somewhat different formulation in Ref. [9]. For small $U_{b f}$ one simply obtains a superfluid of $b$ bosons coexisting with a Fermi liquid of $f$ fermions, while for intermediate $U_{b f}$ and additional Fermi liquid of $c$ bound states forms. Decreasing $U_{b f}$ from large values then leads to a first transition where simultaneously an $f$ Fermi liquid is emerges (at the expense of depleting the $c$ Fermi liquid) and $b$ superfluidity sets in, while at a second transition the $c$ Fermi liquid disappears. Hence, in the fermionic sector there are two standard Lifshitz transitions and no Fermi-surface criticality in this case.

The second case is more interesting: For small $U_{b b}$ one again finds a superfluid of $b$ bosons coexisting with a Fermi liquid; for large $U_{b f}$ the latter can be understood as a Fermi liquid of $c$ composite particles living in a background of sites with single $b$ occupancy. Interestingly, upon decreasing $U_{b b}$ from the Mott insulator, there is now a single transition where $b$ superfluidity sets in. This transition is crossed at fixed bosonic density because the $f$ fermions are invariably bound to bosonic holes due to the large $U_{b f}$. Altman and coworkers have analyzed the corresponding critical field theory, consisting of a condensate order parameter $\phi$ density-coupled to $c$ fermions, in some detail. To leading order, the critical behavior is that of a standard $\phi^{4}$ theory (small logarithmic corrections will either induce a first-order transition or a superfluid instability, but this can be ignored at not too low temperatures). The exciting aspect now is that the physical $f$ fermions are composite objects in terms of the low-energy degrees of freedom, namely the $\phi$ condensate and the $c$ fermions. Then, superfluid criticality induces criticality of the $f$ fermions along the entire Fermi surface. Indeed, the $f$ spectral function at the Fermi wavevector follows $A\left(\vec{k}_{F}, \omega\right) \propto \omega$ at the transition (implying a power-law pseudogap in the momentum-integrated density of states), while $A\left(\vec{k}_{F}, \omega\right)$ displays a sharp delta peak (gap) at small (large) $U_{b b}$.

Thus, the model (1) provides an example for a much sought metallic state with a critical Fermi surface in $d=3$, which occurs here at a superfluid-Mott quantum phase transition due to the formation of bound states between fermionic particles and bosonic holes. Considering the formal similarities of the present setting to slave-particle decompositions employed for fermionic Mott insulators - note that the physical atoms $b$ and $f$ have now taken the roles of the slave particles - one can ask how far these analogies can be driven, i.e., whether models like Eq. (1) can be 
employed as slave-particle quantum simulators [10]. However, the most important difference is that no gauge fields appear in the analysis of Eq. (1), in contrast to slave-particle decompositions. In addition, spin degrees of freedom are absent from the present model (although those might be added, $\left.f_{i} \rightarrow f_{i \sigma}\right)$. Therefore, the physics of the fermionic Hubbard model at the Mott transition is clearly more involved than that of Eq. (1). Nevertheless, it is an interesting starting point to understand states with a quantum critical Fermi surface and, possibly, even more exotic states.

\section{References}

[1] N. F. Mott, Metal-Insulator Transitions (Taylor and Francis, London, 1990).

[2] F. Gebhardt, The Mott Metal-Insulator Transition (Springer, Berlin, 1997).

[3] T. Senthil, Phys. Rev. B 78, 035103 (2008).

[4] Y. Kurosaki, Y. Shimizu, K. Miyagawa, K. Kanoda, and G. Saito, Phys. Rev. Lett. 95, 177001 (2005).

[5] P. A. Lee, N. Nagaosa, and X.-G. Wen, Rev. Mod. Phys. 78, 17 (2006).

[6] S. A. Kivelson, D. S. Rokhsar, and J. P. Sethna, Phys. Rev. B 35, 8865 (1987).

[7] E. Altman, E. Demler, and A. Rosch, preprint arXiv:1205.4026 (2012).

[8] M. Lewenstein, L. Santos, M. Baranov, and H. Fehrmann, Phys. Rev. Lett. 92, 050401 (2004).

[9] S. Powell, S. Sachdev, and H.-P. Büchler, Phys. Rev. B 72, 024534 (2005).

[10] A. Eckardt and M. Lewenstein, Phys. Rev. A 82, 011606 (2010). 